\title{
The Dermatological Clinic in a Leprosy Control Scheme: 10 Years' Experience in Malawi
}

\author{
FRANS RAMPEN* \\ LEPRA Control Project, P.O. Box 496, Blantyre, Malawi
}

\begin{abstract}
A few years after starting a leprosy control scheme in the southern part of Malawi, it became clear that increasing numbers of patients with general dermatological conditions were being referred to the leprologist for diagnosis and treatment. Weekly clinics were therefore established in the 2 urban centres of the control area, and between 1968 and 1975 over 9000 new patients were seen. This paper describes the main conditions diagnosed during these years, listing those of one recent year (1975) in detail. Many curable conditions were seen and the clinics were also a valuable source of new cases of leprosy. The role of the general skin clinic within a leprosy control programme (and in a country with nearly 5 million people and no dermatologist) is discussed, and it is concluded that it may be of considerable diagnostic, therapeutic and research value.
\end{abstract}

\section{Introduction}

From the start of the LEPRA control scheme in the Southern Region of Malawi in 1965, many patients attended the main centre in Blantyre to seek advice and treatment for a wide variety of skin diseases. Initially, most of these patients were referred by medical officers of the adjacent Queen Elizabeth Central Hospital or other hospitals in the vicinity of Blantyre, usually to rule out leprosy. In other instances, patients came from afar, often on their own initiative, and the number of patients attending the centre soon reached such proportions as to warrant the commencement of special dermatological clinics.

From 1968 onwards, skin clinics were held once a week in Blantyre and once a month in Zomba. Leprosy patients, if they came from within the project area, were charted; all clinical and bacteriological particulars were recorded on their cards. They were then issued with the first supply of dapsone and referred to the mobile treatment unit nearest to their own village. Patients with leprosy from outside the project area were given a letter to the medical assistant of the nearest health centre and advice was given as to the type of leprosy and the dosage and length of treatment.

Dermatological cases were diagnosed and treated accordingly. Routine investigations, including skin biopsies, scrapings for fungus infections, urine examination (Wood's light) for porphyria, serological tests for syphilis and

Received for publication 12 January, 1978.

* Present address: Brittenburg 26, Dordrecht, Netherlands. 
skin snips for onchocerciasis were performed during the out-patient attendance and interpreted as rapidly as possible in order to minimize repeated visits.

Reviewing a total of 1508 patients attending the skin clinics in a 12-month period 1970-1971, Worth (1972) listed the main dermatological diagnoses, and Rampen (1976) published a more detailed account of 2664 diagnoses made at the same clinics. Moreover, Rampen(1976) drew particular attention to the frequency of onchocerciasis and its geographical distribution in Malawi.

\section{Findings}

From 1968-1975 over 9000 new skin patients were seen at the dermatological clinics in Blantyre and Zomba. The male:female ratio was $1.7: 1$. The male preponderance is readily explained by the population distribution of the industrial city of Blantyre-Limbe. Most of our skin patients come from this urban area with a population which is chiefly composed of unattached adult males. In the relatively large number of patients who were requested to report back, the response was poor; the average number of attendances per patient over a sustained period of 12 months was 2.3. About $50 \%$ of those needing regular supervision attended only once. Patients with chronic dermatoses like acne vulgaris, atopic eczema and psoriasis also had a poor follow-up, ranging from 2.7 to 3.2 attendances over a 1-year period.

Among the dermatoses most commonly encountered were impetigo, tinea versicolor and other superficial fungus infections, and scabies. The Thyolo district constitutes part of a large onchocerciasis endemic focus and many cases were seen, not only at the skin clinics but also attending the mobile teams.

The drawbacks of a modern society are clearly illustrated by the high incidence of fixed drug eruptions caused by phenolphthalein-containing laxatives, and by the frequent occurrence of vaseline dermatitis, a pustular folliculitis with atrophy, predominantly involving the shins, which is caused by the repeated applications of vaseline creams for cosmetic purposes. At the end of the project it was also noticed that many patients presented with eczematous rashes confined to the exposed parts of the skin. Among other things, the use of soap was incriminated for this phenomenon since Malawians often apply soap after the bath, leaving it on the skin as a cosmetic. Apart from provoking irritant contact dermatitis, some of the locally available soaps were clearly causing a photosensitivity reaction, possibly because they contained halogenated salicylanilides.

A list of skin diseases seen at the clinics during 1975 is shown in Table 1.

Figure 1 shows the numbers of leprosy cases which were registered during the project, and the new dermatological patients seen at the skin clinics from 1968-1975. Whereas the numbers of leprosy patients showed a clear decline, the attendance of skin patients rose considerably. The total numbers of skin attendances (new cases and return visits) averaged 3300 per year over the last 3 years of the project.

Of the leprosy patients seen at the skin clinics (1399 cases), $65 \%$ came from 
TABLE 1

Analysis of skin cases seen during 1975

Infections and infestations

Leprosy
"Observation"
Impetigo, sycosis
Ulcers
Tuberculosis cutis
Verrucae
Other virus infections
Tinea
Candidiasis
Athlete's foot
Scabies
Insect bites
Onchocerciasis
Other inf ections

Dermatitis

Atopic eczema

Seborrhoic eczema

Nonspecific eczema

Neurodermatitis

Contact dermatitis

Pityriasis alba

Vaseline dermatitis

Skinlightener dermatitis

Other eczemas

\section{Papulosquamous eruptions}

Psoriasis

Lichen planus

Palmoplantar keratoses

Follicular keratoses

Other keratoses

\section{Cutaneovascular disorders}

Urticaria 36

Fixed drug eruptions $\quad 29$

Photosensitivity reactions 28

Other erythemas and

drug rashes

\section{Miscellaneous}

Lupus erythematosus $\quad 18$

Granuloma annulare 2

Pityriasis rosea 31

Pellagra 39

Other avitaminoses $\quad 14$

Pemphigus 3

Alopecia areata $\quad 11$

Vitiligo 34

Pigment disturbances $\quad 6$

Acne vulgaris 24

Miliaria 9

Nail disorders, chronic
paronychia

Naevi 13

Haem/lymphangiomata 5

Kaposi's sarcoma $\quad 7$

Other malignant tumours 4

Syphilis 5

Herpes genitalis 7

Condylomata acuminata 4

Other ano-genital conditions $\quad 15$

Pruritus 19

Other skin diseases $\quad 41$

No diagnosis made $\quad 100$

TOTAL $\quad 1608$

within the project area. Over the 8 years of the study this figure ranged from $53 \%-76 \%$ without showing a definite trend.

Figure 2 represents the downward trend of all leprosy cases, registered in the project area from 1968-1975, against the number of leprosy cases, who were recorded at the dermatological clinics, i.e. patients who came from the project area only. Whereas the annual totals of leprosy cases in the whole area show a progressive decline, the numbers registered at the skin clinics are more or less stable. The relative importance of the skin clinics for the diagnosis of leprosy is demonstrated in Fig. 3: the percentage of leprosy patients diagnosed at the clinics as compared to the cases registered in the whole area increased from $6.7 \%$ in 1968 to $26.1 \%$ in 1975 .

The annual totals of new skin patients increased steadily since 1968 , whereas the numbers of all leprosy patients diagnosed at the clinics-from within and outside the project-remained stable (Fig. 4). Consequently the 
percentages of leprosy cases in proportion to the total numbers of skin patients dropped from $26.0 \%$ in 1968 to $10.6 \%$ in 1975 (Fig. 5).

\section{Discussion}

Since the total attendances at our skin clinics increased substantially from 1968-1975, the work load increased correspondingly and at the end of the LEPRA project it was estimated that $15-20 \%$ of the working hours of the senior medical staff was spent on dermatology. Although the ratio of leprosy

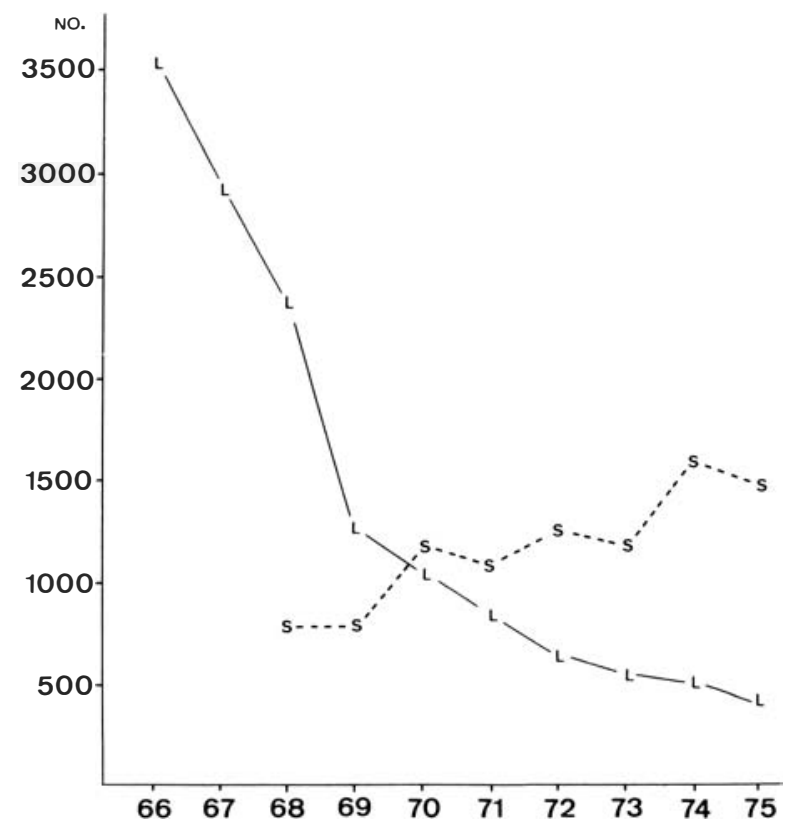

Fig. 1. Leprosy cases (L) registered during the 10 years of the project, compared with new skin patients (S) attending the dermatological clinics from 1968-1975.

cases diagnosed at the clinics dropped in relation to the total number of skin attendances, the relative importance of the skin clinics in proportion to the total of leprosy patients registered in the whole area gradually increased over the years of the study. From this point of view, the skin clinics, though time consuming, served their purpose.

The dermatoses constitute a high proportion of the total morbidity in tropical countries. However, the mortality rate is negligible. Nevertheless, conditions like tuberculosis of the skin, syphilis, erythroderma, pemphigus, malignant melanoma, Kapsi's sarcoma, etc., require early recognition and adequate management. It is also clear that the collection of these data is potentially important to research on infective, parasitic and malignant disease 


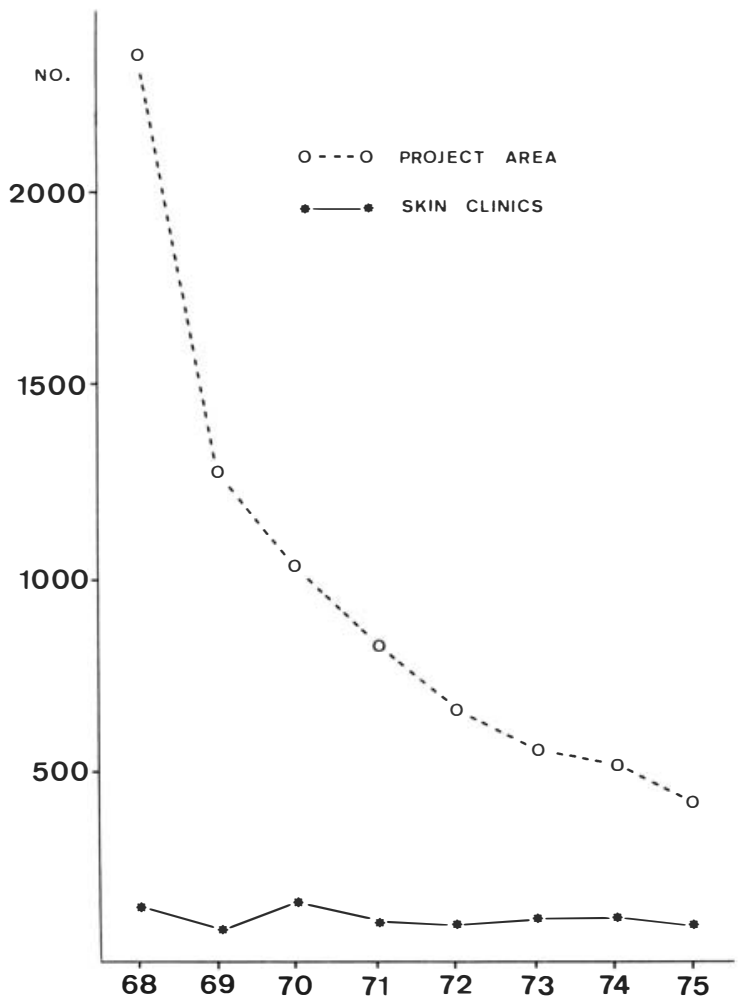

Fig. 2. Leprosy cases registered in the whole project area, compared with the cases first diagnosed at the skin clinics (1968-1975).

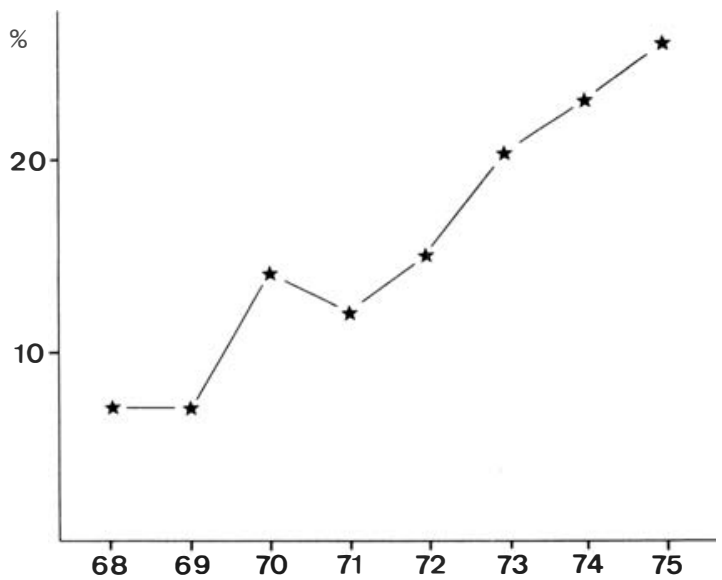

Fig. 3. Percentages of leprosy instances, coming from within the project area and first diagnosed at the skin clinics, proportional to the total number of leprosy padtients)registered. StUdOS 


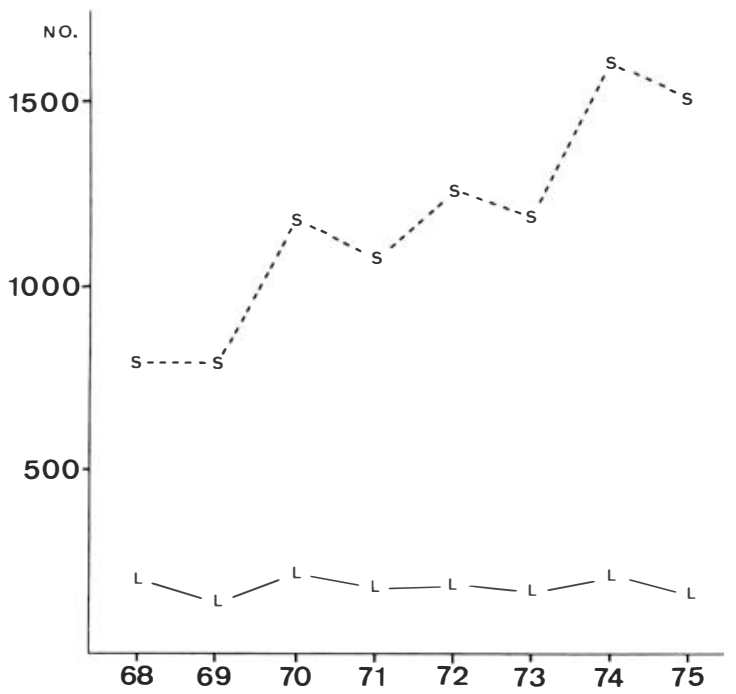

Fig. 4. Annual totals of new skin patients (S), compared with all leprosy cases (L) attending the dermatological clinics.

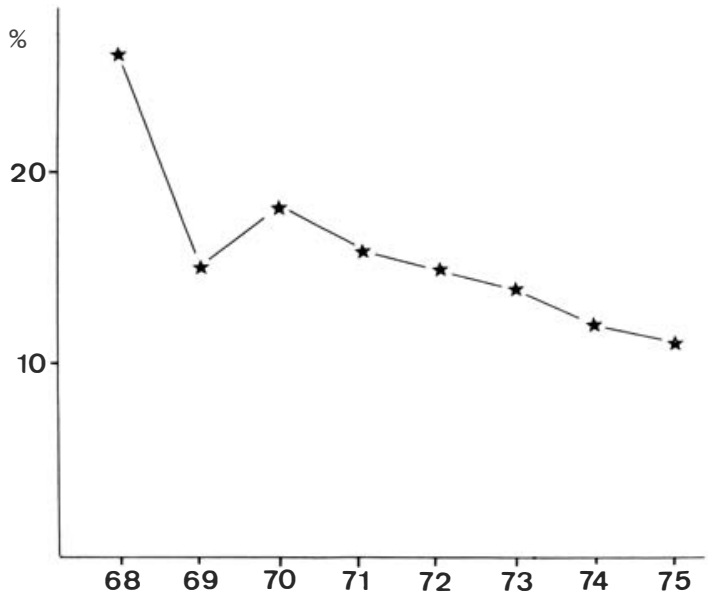

Fig. 5. Percentages of leprosy cases (in and outside area combined) seen at the skin clinics, proportional to the annual totals of new skin attendances. 
in Africa. Dermatology, for obvious reasons, is not a priority of the health ministries of developing countries; in the whole of tropical Africa, there are fewer dermatologists than in cities like Glasgow or Amsterdam. The duties of the leprologist in an endemic area of Africa (and many other countries) entail a knowledge of clinical medicine, neurology, administration and practical psychology, but he can hardly avoid acquiring a vast experience in the field of dermatology also. The establishment of skin clinics, at the urban centres of this leprosy control scheme in Malawi, seems to have been of considerable diagnostic and therapeutic benefit to a large number of people, and has in addition attracted a significant number of new patients with leprosy.

\section{Acknowledgement}

I am grateful to Dr B. D. Molesworth, Director of LEPRA, Malawi, for permission to publish.

\section{References}

Rampen, F. (1976). The spectrum of dermatology in Malawi. E. Afr. med.J. 53, 398.

Rampen, F. (1976). The geographical distribution of onchocerciasis in Malawi. E. Afr. med. J. 53, 251 .

Worth, R. W. (1972). Leprosy controlled? Oxf. med. Sch. Gaz. 24 (3), 41 